OPEN ACCESS

Edited by:

Xueming Liu,

Zhejiang University, China

Reviewed by:

Aiping Luo,

South China Normal University, China

Han Zhang,

Shenzhen University, China

${ }^{*}$ Correspondence:

Xiaohui L

lixiaohui@snnu.edu.cn

Yani Zhang

yanizhang1@163.com

Specialty section: This article was submitted to

Optics and Photonics,

a section of the journal

Frontiers in Physics

Received: 26 July 2019 Accepted: 19 September 2019

Published: 04 October 2019

Citation:

Shi Z, Li X, Zhang Y, Li H, Zhao Y, Guo $P$ and Guo Y (2019) Graphdiyne for Ultrashort Pulse Generation in an Erbium-Doped Hybrid Mode-Locked

Fiber Laser. Front. Phys. 7:150.

doi: 10.3389/fphy.2019.00150

\section{Graphdiyne for Ultrashort Pulse Generation in an Erbium-Doped Hybrid Mode-Locked Fiber Laser}

\author{
Zhaojiang Shi ${ }^{1,2}$, Xiaohui Li $^{1,2 *}$, Yani Zhang ${ }^{3,4 *}$, Hongqi Li $^{1,2}$, Yang Zhao ${ }^{1,2}$, Penglai Guo ${ }^{1,2}$ \\ and Yixuan Guo ${ }^{1,2}$
}

${ }^{1}$ College of Physics and Information Technology, Shaanxi Normal University, Xi'an, China, ${ }^{2}$ National Demonstration Center for Experimental X-Physics Education, Shaanxi Normal University, Xi'an, China, ${ }^{3}$ School of Physics and Optoelectronics Technology, Baoji University of Arts and Science, Baoji, China, ${ }^{4}$ Baoji Engineering Technology Research Centre on Ultrafast Laser and New Materials, Baoji, China

An erbium-doped hybrid passively mode-locked fiber laser based on few-layer graphdiyne (GDY) saturable absorber (SA) has been investigated for the first time. Hybrid mode-locked fiber laser is composed of non-linear polarization rotation (NPR) technology and GDY-SA. The central wavelength, pulse width and repetition rate of the output pulse are $1530.7 \mathrm{~nm}, 690.2 \mathrm{fs}$ and $14.7 \mathrm{MHz}$, respectively. Compared with the passively mode-locked pulse laser with GDY-SA or NPR technology alone, the output pulse width of hybrid passively mode-locked fiber laser is reduced more than $50 \mathrm{fs}$. It is demonstrated that the performance of GDY can be potentially applied in ultrafast laser.

Keywords: graphdiyne, saturable absorber, mode-locked, fiber laser, ultrafast photonics

\section{INTRODUCTION}

Ultra-short pulse mode-locked fiber laser has been extensively investigated due to its applications in laser physics, material science, nanotechnology and biomedicine, etc. This type of laser is simple, compact, and easy to integrate, allowing for high quality pulse [1-8]. In recent years, passively mode-locked fiber lasers have gained more development than active mode-locked fiber lasers. The use of SA to obtain ultrashort pulses is becoming the mainstream of passively mode-locked fiber lasers [9-13]. More and more two-dimensional (2D) materials with outstanding optical properties have been applied in the field of lasers [14-18]. These materials are significantly characterized by adjustable non-linear absorption coefficient, short relaxation time and recovery time, and low optical loss [19-21]. Therefore, these materials have been widely used in ultrafast photonics to obtain high power and broadband spectrum mode-locked pulse sources [22-38], such as semiconductor saturable absorption mirror (SESAM), single-walled carbon nanotube (SWCNT), graphene, graphene oxide, topological insulators (TI), black phosphorus (BP), bismuthine, $\mathrm{WS}_{2}$, etc. In 2010, Popa et al. reported a new passively mode-locked fiber laser, which uses graphene as SA and generates ultrashort pulses with a pulse width of $>200$ fs [34]. In 2013, an all-fiber all-normal dispersion passively mode-locked $\mathrm{Yb}$-doped ring laser based on graphene oxide was reported [35]. In 2018, Yang et al. obtained high energy rectangular pulse on the basis of $\mathrm{WS}_{2}$ cladding microfiber SA [36]. Very recently, GDY has been investigated, whose property is similar to other as carbon based materials, such as CNT, graphene and fullerene [39-45]. Furthermore, the bandgap of GDY 


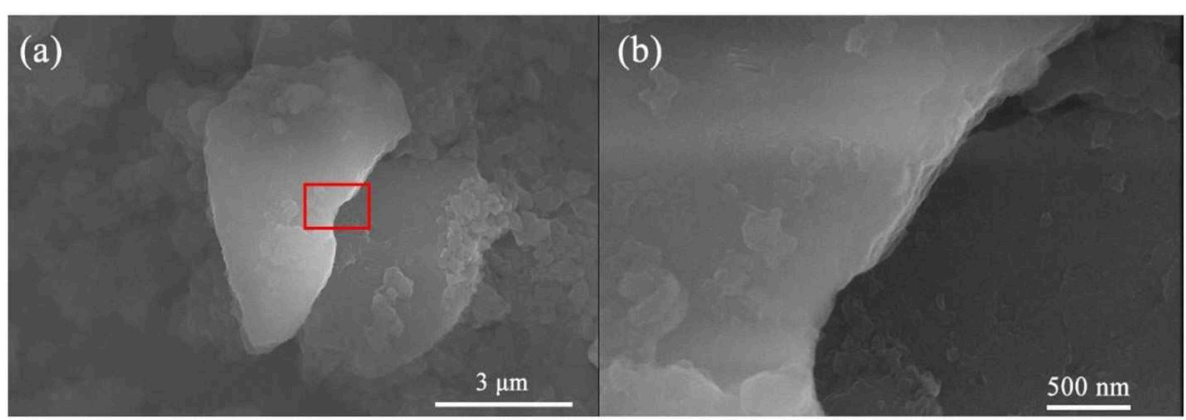

FIGURE 1 | The SEM image of GDY powder in chlorobenzene solution with different magnification. (a) The magnification of $3 \mu \mathrm{m}$. (b) The magnification of 500 nm.

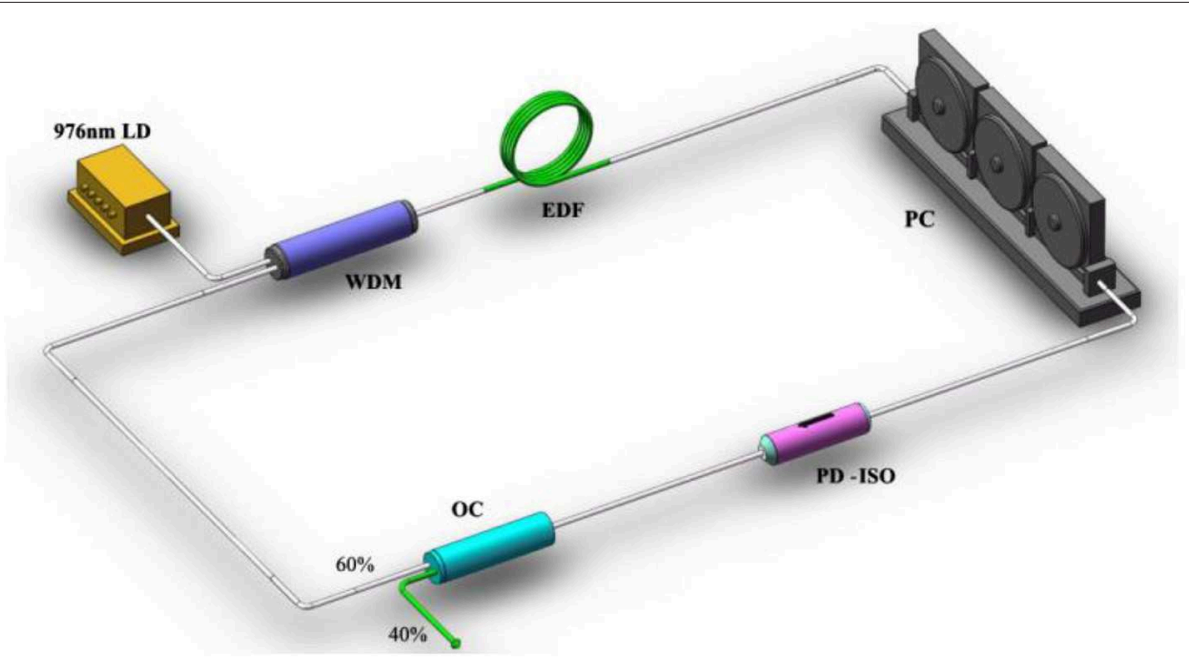

FIGURE 2 | Experimental setup of the NPR mode-locked erbium-doped fiber laser.

is around $0.52 \mathrm{eV}$, which should have excellent performance in near infrared regime. In order to prepare this material, several groups have demonstrated a methodology to generate networks of combinations of $\mathrm{sp}$ and $\mathrm{sp}^{2}$, or $\mathrm{sp}$ and $\mathrm{sp}^{3}$ hybridized carbons. In addition, the carbon elements inside GDY are found to be highly conjugated carbon rich organic molecules in the process of synthesis. Due to its special electrical and optical properties, Zhao et al. have applied GDY to a $1.5 \mu \mathrm{m}$ passively mode-locked fiber laser [46]. It is well known that GDY has broad application prospects in the fields of non-linear photonics and ultrafast photonics.

Based on the previous work, a hybrid mode-locked erbiumdoped fiber laser is fabricated by using NPR technology and GDY-SA prepared by spin-coating method. The hybrid modelocked erbium-doped fiber laser can obtain a stable mode-locked pulse with a central wavelength of $1530.7 \mathrm{~nm}$ and a pulse width of 690.2 fs. Compared with the mode-locked pulse obtained by GDY-SA or NPR technology alone, its performance can be improved significantly and the pulse width can be reduced by nearly tens of fs. It is proved that GDY has potential application prospects in the field of ultrafast lasers and other non-linear optical elements.

\section{CHARACTERIZATION OF GDY AND PREPARATION OF GDY-SA THIN FILMS}

Figure 1a shows a scanning electron microscope (SEM) image of GDY powder in a chlorobenzene solution at a magnification of $3 \mu \mathrm{m}$, from which it can be seen that GDY has a very good planar structure. Figure $\mathbf{1 b}$ is the SEM image of the GDY powder in the chlorobenzene solution at the red frame in Figure 1a enlarged at $500 \mathrm{~nm}$. As can be seen from the graph, the edges of GDY have stratification and obvious continuity.

GDY-SA thin films were prepared by spin-coating at different rotational speeds. Firstly, $1 \mathrm{mg}$ GDY and $1 \mathrm{~mL}$ chlorobenzene solution were mixed with ultrasound for $10 \mathrm{~h}$. Then, the obtained mixed solution and polymethyl methacrylate (PMMA) colloid were ultrasonicated at a ratio of $1: 2$ for $10 \mathrm{~h}$, and then placed in a spinning machine to obtain a GDY-SA film. The coating 

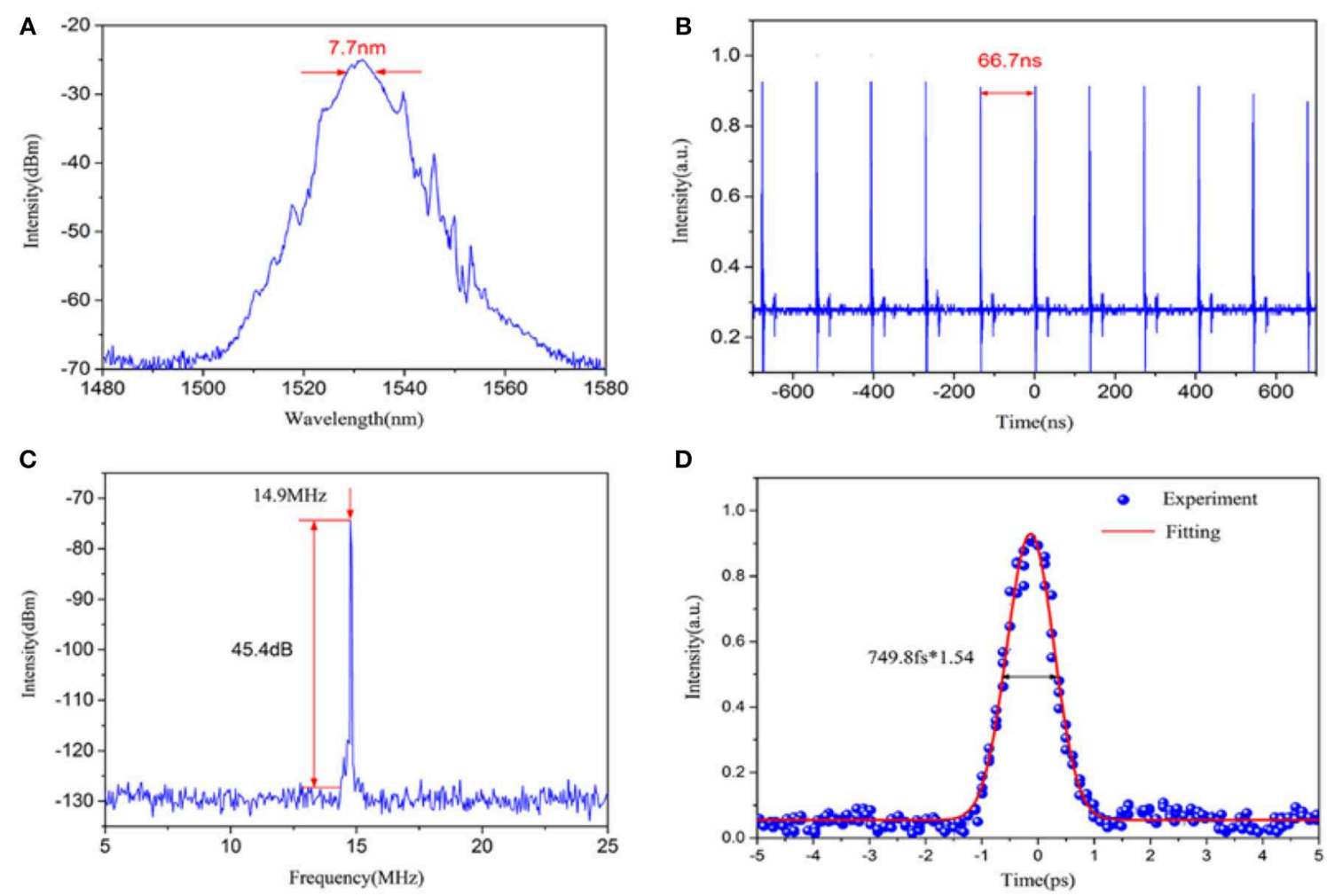

FIGURE 3 | Pulse output characteristics of NPR mode-locked laser when the pump power is $135 \mathrm{~mW}$. (A) Output spectrum, (B) pulse trace on oscilloscope, (C) RF spectrum, (D) autocorrelation trace.

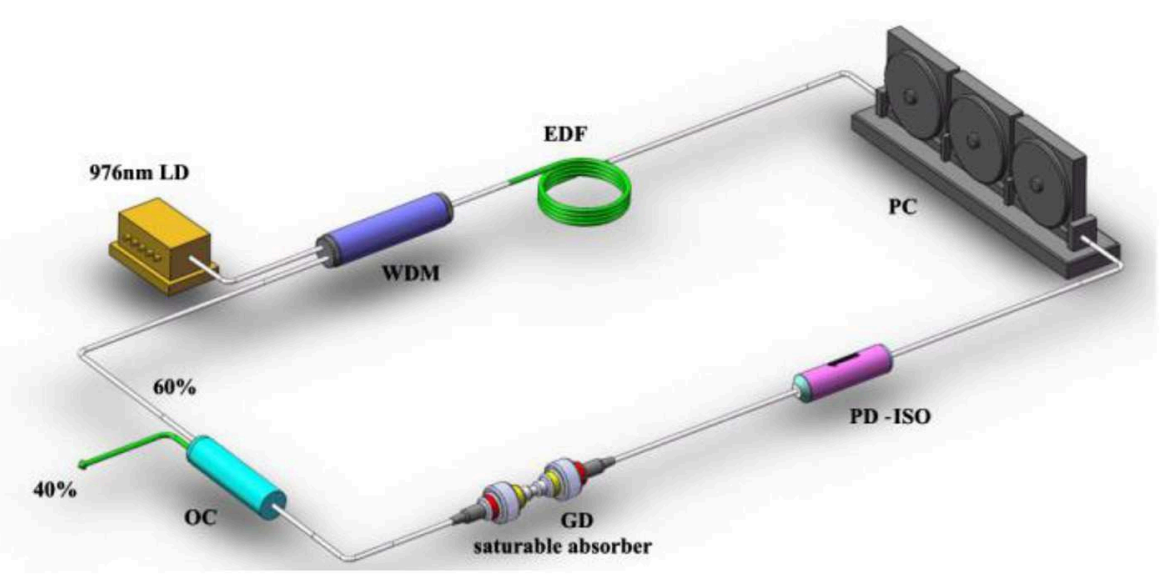

FIGURE 4 | Experimental setup of the hybrid erbium-doped mode-locked fiber laser.

was rotated using three revolutions of $1,000,1,200$, and 1,500 $\mathrm{rad} / \mathrm{min}$,respectively. Finally, the obtained film was dried in a dry box for $1 \mathrm{~h}$. After many experiments, it was found that a high quality GDY-SA film was prepared at a spin speed of $1,000 \mathrm{rad} / \mathrm{min}$. Combined with the high stability characteristics of GDY itself, it can be proved that GDY-SA in this experiment has good stability and can withstand long-time illumination without change.

\section{EXPERIMENTAL SETUP AND RESULTS}

\section{NPR Mode-Locked Erbium-Doped Fiber} Laser

Passively mode-locked erbium-doped fiber laser based on NPR, as shown in Figure 2, is mainly composed of the lasing diode (LD) with the output wavelength of $976 \mathrm{~nm}$, wavelength division multiplexing (WDM), single-mode fiber (SMF), erbium-doped 


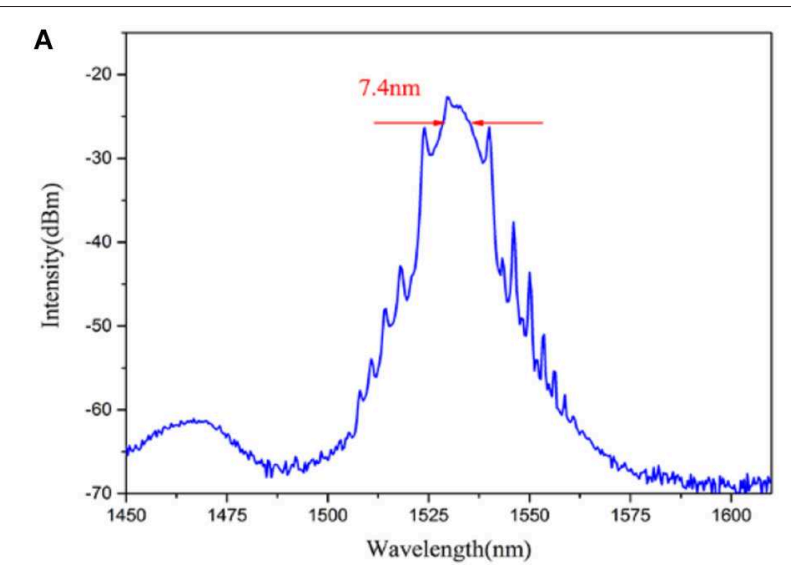

C

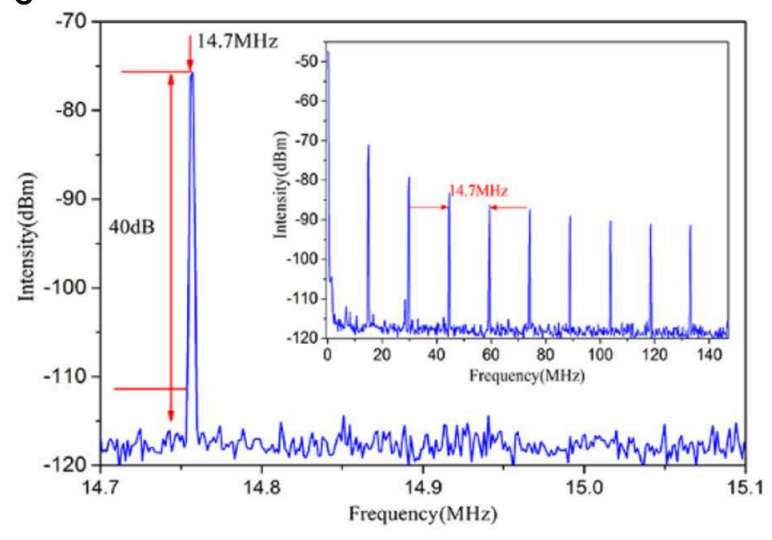

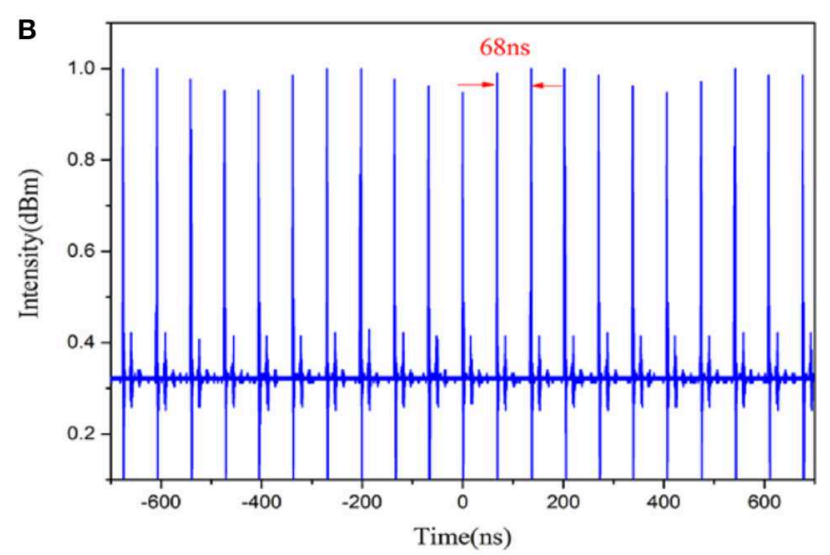

D

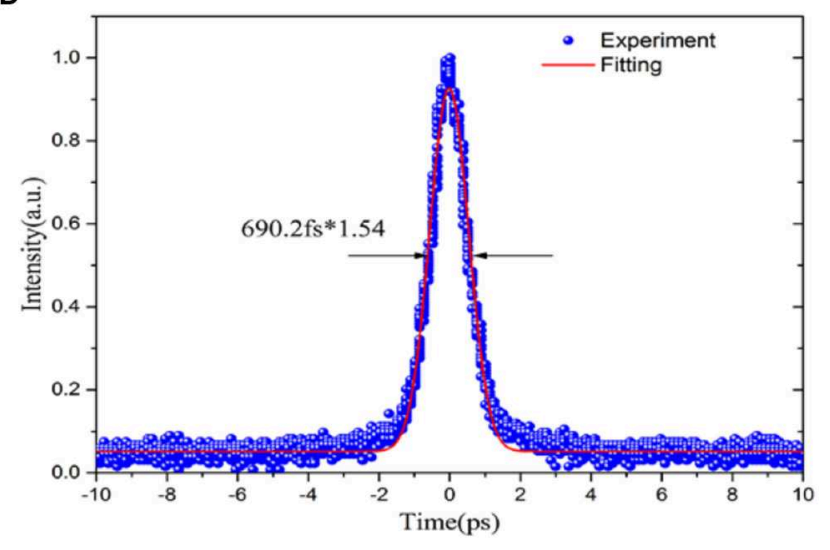

FIGURE 5 | Pulse output characteristics of hybrid mode-locked laser when the pump power is 135 mW. (A) output spectrum, (B) pulse trace on oscilloscope, (C) RF spectrum, (D) autocorrelation trace.

fiber (EDF), polarization controller (PC), polarization dependent isolator (PD-ISO), and optical coupler (OC). The output ratio of OC is $40: 60$. The length of EDF is $0.75 \mathrm{~m}$ and the total cavity length of laser is $14.3 \mathrm{~m}$.

After increasing the pump power to $135 \mathrm{~mW}$, the stable mode-locked pulse output can be obtained by adjusting the PC. The output spectrum is shown in Figure 3A, with a central wavelength of $1532.1 \mathrm{~nm}$ and a $3-\mathrm{dB}$ spectral width of $7.7 \mathrm{~nm}$. The output pulse trace is shown in Figure $\mathbf{3 B}$ with a pulse interval of $66.7 \mathrm{~ns}$. The corresponding radio frequency (RF) spectrum is shown in Figure 3C, with a signal-to-noise ratio (SNR) of 45.4 $\mathrm{dB}$ and a repetition rate of 14.9 MHz. As shown in Figure 3D, the pulse width measured by the autocorrelator is $749.8 \mathrm{fs}$.

\section{Hybrid Mode-Locked Erbium-Doped Fiber Laser}

As shown in Figure 4, a hybrid erbium-doped mode-locked fiber laser is formed by adding GDY-SA to the previous NPR erbiumdoped mode-locked fiber laser. The total cavity length increased slightly from the previous one to $14.6 \mathrm{~m}$. Under the combined action of NPR technology and GDY-SA, the pulses are further compressed and finally output via OC.

When the pump power is $135 \mathrm{~mW}$, a stable mode-locked pulse output can be obtained. The highest threshold for this experiment was $210 \mathrm{~mW}$. However, this is due to the threshold of the maximum power of the $\mathrm{LD}$, and does not rule out the possibility that the actual threshold is higher. The output spectrum is shown in Figure 5A, with a central wavelength of $1530.7 \mathrm{~nm}$ and a 3$\mathrm{dB}$ spectral width of $7.4 \mathrm{~nm}$. It can be found that the spectrum has a large change with the spectrum in Figure 3A, which is due to the action of GDY-SA. The output pulse trace is shown in Figure 5B with a pulse interval of 68 ns. The corresponding $\mathrm{RF}$ spectrum is shown in Figure 5C, with a signal-to-noise ratio (SNR) of $40 \mathrm{~dB}$ and a repetition rate of $14.7 \mathrm{MHz}$, and it can be seen from the illustration that the pulse is relatively stable. As shown in Figure 5D, the pulse width measured by the autocorrelator is 690.2 fs. And the maximum pulse energy is $0.4 \mathrm{~nJ}$. The pulse sequence and spectral output for 2 days were basically stable and could work in a stable state for a long time.

\section{CONCLUSION}

In conclusion, a hybrid erbium-doped fiber mode-locked laser based on GDY-SA and NPR technology has been successfully realized. The band gap of GDY is about $0.52 \mathrm{eV}$. This direct band gap enables GDY to be used as a SA in ultrafast photonics. After adding GDY-SA, the output pulse width of hybrid erbium-doped 
fiber mode-locked laser is reduced to $690.2 \mathrm{fs}$, which proves that GDY-SA plays an important role in hybrid erbium-doped fiber mode-locked laser. The results show that GDY-SA can be used as an excellent optical modulation material in ultrafast photonics in the infrared wavelength range.

\section{DATA AVAILABILITY STATEMENT}

All datasets generated for this study are included in the manuscript/supplementary files.

\section{AUTHOR CONTRIBUTIONS}

ZS and HL wrote the manuscript. XL, YZhang, YZhao, PG, and YG revised the text. All the authors contributed conception and design of the study.

\section{REFERENCES}

1. Du J, Wang QK, Jiang GB, Xu CW, Zhao CJ, Xiang YJ, et al. Ytterbiumdoped fiber laser passively mode locked by few-layer Molybdenum Disulfide $\left(\mathrm{MoS}_{2}\right)$ saturable absorber functioned with evanescent field interaction. Sci Rep. (2014) 4:7. doi: 10.1038/srep06346

2. Mao D, Zhang SL, Wang YD, Gan XT, Zhang WD, Mei T, et al. WS $\mathrm{S}_{2}$ saturable absorber for dissipative soliton mode locking at 1.06 and $1.55 \mu \mathrm{m}$. Opt Express. (2015) 23:27509-19. doi: 10.1364/OE.23.027509

3. Liu J, Wu SD, Yang QH, Wang P. Stable nanosecond pulse generation from a graphene-based passively Q-switched Yb-doped fiber laser. Opt Lett. (2011) 36:4008-10. doi: 10.1364/OL.36.004008

4. Liu X, Yao X, Cui Y. Real-time observation of the buildup of soliton molecules. Phys Rev Lett. (2018) 121:023905. doi: 10.1103/PhysRevLett.121.023905

5. Wang ZQ, Zhan L, Wu J, Zou ZX, Zhang L, Qian K, et al. Self-starting ultrafast fiber lasers mode-locked with alcohol. Opt Lett. (2015) 40:3699-702. doi: 10.1364/OL.40.003699

6. Dianov EM. Bismuth-doped optical fibers: a challenging active medium for near-IR lasers and optical amplifiers. Light-Sci Appl. (2012) 1:e12. doi: 10.1038/lsa.2012.12

7. Sedov ES, Rubo YG, Kavokin AV. Polariton polarization rectifier. Light-Sci Appl. (2019) 8:79. doi: 10.1038/s41377-019-0189-Z

8. Limpert J, Stutzki F, Jansen F, Otto HJ, Eidam T, Jauregui C, et al. Yb-doped large-pitch fibres: effective single-mode operation based on higher-order mode delocalization. Light-Sci Appl. (2012) 1:e8. doi: 10.1038/lsa.2012.8

9. Liu ZB, He XY, Wang DN. Passively mode-locked fiber laser based on a hollow-core photonic crystal fiber filled with few-layered graphene oxide solution. Opt Lett. (2011) 36:3024-6. doi: 10.1364/OL.36.003024

10. Hui Z, Xu W, Li X, Guo P, Zhang Y, Liu J. $\mathrm{Cu}_{2} \mathrm{~S}$ nanosheets for ultrashort pulse generation in the near-infrared region. Nanoscale. (2019) 11:6045-51. doi: 10.1039/C9NR00080A

11. Li XH, Yu XC, Sun ZP, Yan ZY, Sun B, Cheng YB, et al. High-power graphene mode-locked $\mathrm{Tm} / \mathrm{Ho}$ co-doped fiber laser with evanescent field interaction. Sci Rep. (2015) 5:8. doi: 10.1038/srep16624

12. Zhang H, Lu SB, Zheng J, Du J, Wen SC, Tang DY, et al. Molybdenum disulfide $\left(\mathrm{MoS}_{2}\right)$ as a broadband saturable absorber for ultra-fast photonics. Opt Express. (2014) 22:7249-60. doi: 10.1364/OE.22.007249

13. Sun $\mathrm{ZH}$, Chang HX. Graphene and graphene-like two-dimensional materials in photodetection: mechanisms and methodology. ACSNano. (2014) 8:413356. doi: 10.1021/nn500508c

14. Xu MS, Liang T, Shi MM, Chen HZ. Graphene-like two-dimensional materials. Chem Rev. (2013) 113:3766-98. doi: 10.1021/cr300263a

15. Carvalho, Ribeiro RM, Neto AHC. Band nesting and the optical response of two-dimensional semiconducting transition metal dichalcogenides. Phys Rev B. (2013) 88:6. doi: 10.1103/PhysRevB.88.115205

\section{FUNDING}

This research was supported by the National Natural Science Foundation of China (61605106); Funded projects for the Academic Leader and Academic Backbones, Shaanxi Normal University (18QNGG006); Starting Grants of Shaanxi Normal University (Grant No. 1112010209, 1110010717); Fundamental Research Funds For the Central Universities (GK201802006, 2018CSLY005); Open Research Fund of State Key Laboratory of Pulsed Power Laser Technology, Electronic Engineering Institute (No. SKL2017KF02); Open Fund of State Key Laboratory of Information Photonics and Optical Communications (Beijing University of Posts and Telecommunications), P. R. China (IPOC2017B012); Open Research Fund of State Key Laboratory of Transient Optics and Photonics, Chinese Academy of Sciences (No. SKLST201401, SKLST201809).

16. Li XH, Wang YS, Zhang W, Zhao W. Experimental observation of soliton molecule evolution in Yb-doped passively mode-locked fiber lasers. Laser Phys Lett. (2014) 11:5. doi: 10.1088/1612-2011/11/7/075103

17. Yang G, Liu YG, Wang Z, Lou JC, Wang ZH, Liu ZB. Broadband wavelength tunable mode-locked thulium-doped fiber laser operating in the $2 \mu \mathrm{m}$ region by using a graphene saturable absorber on microfiber. Laser Phys Lett. (2016) 13:5. doi: 10.1088/1612-2011/13/6/065105

18. Jackson SD. Towards high-power mid-infrared emission from a fibre laser. Nat Photonics. (2012) 6:423-31. doi: 10.1038/nphoton.2012.149

19. Mao D, Wang YD, Ma CJ, Han L, Jiang BQ, Gan XT, et al. $\mathrm{WS}_{2}$ mode-locked ultrafast fiber laser. Sci Rep. (2015) 5:7. doi: 10.1038/srep07965

20. Liu X, Han D, Sun Z, Zeng C, Lu H, Mao D, et al. Versatile multi-wavelength ultrafast fiber laser mode-locked by carbon nanotubes. Sci Rep. (2013) 3:2718. doi: $10.1038 /$ srep02718

21. Jung M, Koo J, Debnath P, Song YW, Lee JH. AMode-Locked $1.91 \mu \mathrm{m}$ fiber laser based on interaction between graphene oxide and evanescent field. Appl Phys Express. (2012) 5:3. doi: 10.1143/APEX.5.112702

22. Matte H, Gomathi A, Manna AK, Late DJ, Datta R, Pati SK, etal. $\mathrm{MoS}_{2}$ and $\mathrm{WS}_{2}$ analogues of grapheme. Angew Chem Intc Edit. (2010) 49:4059-62. doi: 10.1002/anie.201000009

23. Liu XM, Popa D, Akhmediev N. Revealing the transition dynamics from QSwitching to mode locking in a soliton laser. Phys Rev Lett. (2019) 123:093901. doi: 10.1103/PhysRevLett.123.093901

24. Ren L, Qi X, Liu YD, Hao GL, Huang ZY, Zou XH, et al. Large-scale production of ultrathin topological insulator bismuth telluride nanosheets by a hydrothermal intercalation and exfoliation route. J Mater Chem. (2012) 22:4921-6. doi: 10.1039/c2jm15973b

25. Splendiani, Sun L, Zhang YB, Li TS, Kim J, Chim CY, et al. Emerging photoluminescence in monolayer MoS2. Nano Lett. (2010) 10:1271-5. doi: $10.1021 / \mathrm{nl} 903868 \mathrm{w}$

26. Zhang H, Virally S, Bao QL, Ping LK, Massar S, Godbout N, et al. Z-scan measurement of the nonlinear refractive index of grapheme. Opt Lett. (2012) 37:1856-58. doi: 10.1364/OL.37.001856

27. Huang PL, Lin SC, Yeh CY, Kuo HH, Huang SH, Lin GR, et al. Stable mode-locked fiber laser based on CVD fabricated graphene saturable absorber. Opt Express. (2012) 20:2460-5. doi: 10.1364/OE.20.0 02460

28. Liu X, Cui Y. Revealing the behavior of soliton buildup in a mode-locked laser. Adv Photon. (2019) 1:016003. doi: 10.1117/1.AP.1.1.016003

29. Cui YD, Lu FF, Liu XM. MoS 2 -clad microfibre laser delivering conventional, dispersion-managed and dissipative solitons. Sci Rep. (2016) 6:8. doi: 10.1038/srep30524

30. Wu XQ, Yu SL, Yang HR, Li WL, Liu XM, Tong LM. Effective transfer of micron-size graphene to microfibers for photonic applications. Carbon. (2016) 96:1114-9. doi: 10.1016/j.carbon.2015.10.069 
31. Wang C, Wang L, Li X, Luo W, Feng T, Zhang Y, et al. Few-layer bismuthene for femtosecond soliton molecules generation in Er-doped fiber laser. Nanotechnology. (2019) 30:025204. doi: 10.1088/1361-6528/aae8c1

32. Cui YD, Liu XM. Revelation of the birth and extinction dynamics of solitons in SWNT-mode-locked fiber lasers. Photonics Res. (2019) 7:423-30. doi: 10.1364/PRJ.7.000423

33. Zhang Y, Li XH, Qyyum A, Feng TC, Guo PL, Jiang J, et al. PbSNanoparticles for Ultrashort Pulse Generation in Optical Communication Region. Part Part Syst Charact. (2018) 35:6. doi: 10.1002/ppsc.201800341

34. Popa D, Sun Z, Torrisi F, Hasan T, Wang F, Ferrari AC. Sub 200 fs pulse generation from a graphene mode-locked fiber laser. Appl Phys Lett. (2010) 97:3. doi: 10.1063/1.3517251

35. Li XH, Wang YG, Wang YS, Zhang YZ, Wu K, Shum PP, et al. Allnormal-dispersion passively mode-locked $\mathrm{Yb}$-doped fiber ring laser based on a graphene oxide saturable absorber. Laser Phys Lett. (2013) 10:075108. doi: 10.1088/1612-2011/10/7/075108

36. Yang HR, Liu XM. WS $\mathrm{W}_{2}$-Clad microfiber saturable absorber for high-energy rectangular pulse fiber laser. IEEEJ Sel Top Quantum Electron. (2018) 24:7. doi: 10.1109/JSTQE.2017.2757142

37. Luo ZC, Luo AP, Xu WC. Tunable and Switchable Multiwavelength Passively Mode-Locked Fiber Laser Based on SESAM and Inline birefringence comb filter. IEEEPhotonics J. (2011) 3:64-70. doi: 10.1109/JPHOT.2010.2102012

38. Lu L, Liang ZM, Wu LM, Chen YX, Song YF, Dhanabalan SC, et al. Few-layer bismuthene: sonochemical exfoliation, nonlinear optics and applications for ultrafast photonics with enhanced stability. Laser Photon Rev. (2018) 12:10. doi: 10.1002/lpor.201700221

39. Zhao GK, Lin W, Chen HJ, Lv YK, Tan XM, Yang ZM, et al. Dissipative soliton resonance in Bismuth-doped fiber laser. Opt Express. (2017) 25:20923-31. doi: 10.1364/OE.25.020923

40. Liu XM, Pang M. Revealing the buildup dynamics of harmonic modelocking states in ultrafast lasers. Laser Photon Rev. (2019) 13:1800333. doi: 10.1002/lpor.201800333
41. Li GX, Li YL, Liu HB, Guo YB, Li YJ, Zhu DB. Architecture of graphdiyne nanoscale films Chem Commun. (2010) 46:3256-8. doi: 10.1039/b92 2733d

42. Jin ZW, Yuan MJ, Li H, Yang H, Zhou Q, Liu HB, et al. Graphdiyne: an efficient hole transporter for stable high-performance colloidal quantum dot solar cells. Adv Funct Mater. (2016) 26:5284-89. doi: 10.1002/adfm.2016 01570

43. He JJ, Bao KJ, Cui WW, Yu JJ, Huang CS, Shen XY, et al. Construction of largearea uniform graphdiyne film for high-performance lithium-ion batteries. Chem Eur J. (2018) 24:1187-92. doi: 10.1002/chem.201704581

44. Zhu Y, Bai HC, Huang YH. Electronic property modulation of onedimensional extended graphdiyne nanowires from a first-principle crystal orbital view. ChemistryOpen. (2016) 5:78-87. doi: 10.1002/open.2015 00154

45. Jiao Y, Du AJ, Hankel M, Zhu ZH, Rudolph V, Smith SC. Graphdiyne: a versatile nanomaterial for electronics and hydrogen purification. Chem Commun. (2011) 47:11843-5. doi: 10.1039/c1cc15129k

46. Zhao Y, Guo P, Li X, Jin Z. Ultrafast photonics application of graphdiyne in optical communication region. Carbon. (2019) 149:336-41. doi: 10.1016/ j.carbon.2019.04.075

Conflict of Interest: The authors declare that the research was conducted in the absence of any commercial or financial relationships that could be construed as a potential conflict of interest.

Copyright (c) 2019 Shi, Li, Zhang, Li, Zhao, Guo and Guo. This is an open-access article distributed under the terms of the Creative Commons Attribution License (CC $B Y)$. The use, distribution or reproduction in other forums is permitted, provided the original author(s) and the copyright owner(s) are credited and that the original publication in this journal is cited, in accordance with accepted academic practice. No use, distribution or reproduction is permitted which does not comply with these terms. 